\title{
Die Grundzüge der Spiritualität des Ordenslebens im sechsten Kapitel in der dogmatischen Konstitution über die Kirche „Lumen gentium“
}

\section{The basic principles of the spirituality of cones crated life as presented in chapter VI of Lumen gentium, the Dogmatic Constitution on the Church}

\begin{abstract}
The article presents the basic principles of the spirituality of consecrated life as they are laid out in chapter VI of the Constitution on the Church. These principles can be summed up as the following:

1. Consecrated life means the imitation of Christ.

2. This imitation is of a charismatic nature, thus can only be lived by vocation, with the power of and originating from the Holy Spirit.

3. The fruits of the works of the Holy Spirit, and consequently, the enforcement of the imitation of Christ is love; a love that is always love towards God and your neighbour. How diverse talents and vocations can be.

4. Thanks to this love, enforced by the Holy Spirit, consecrated life presents itself as a present from Christ to the Church. It is therefore, placed in the centre of Church life and serves the Church not just by obedience and ministry, but even deeper by a living witness that reflects the pilgrimage of a Christian, and the hope for a life to come.
\end{abstract}

\section{Keywords}

Consecrated life, imitation of Christ, vocation, evangelical counsels. 


\section{Nachfolge Christi}

Im ersten Satz des Kapitels 6 der Kirchenkonstitution heißt es, dass „die evangelischen Räte (...) in Wort und Beispiel des Herrn begründet "1 ${ }^{\text {"1 }}$ sind. Es wird jedem sofort einleuchten, dass hiermit eine entscheidende Behauptung aufgestellt ist. Unsere Zeit ist dadurch gekennzeichnet, dass man alle Vorstellungen auf ihren Wirklichkeitsgehalt hin überprüft. Selbst bei den geistigen Wirklichkeiten geht der heutige Mensch fast wie ein Naturwissenschaftler vor, der durch das Experiment die Dinge möglichst so, wie wir ihre Wirkung erfahren, erreichen will. Allem, was sich nicht durch Erfahrung als real erweisen lässt, steht der Mensch von heute skeptisch gegenüber. In der Wirklichkeit des Glaubens führt diese Haltung dazu, dass alles auf seinen Ursprung hin geprüft und mit der Wurzel des Glaubens, mit der Person Christi und dem Evangelium, konfrontiert wird. ${ }^{2}$ So kommt es zur Kritik vor allem der jungen Christen an so manchem überlieferten Brauch. Die Frage, die man stellt, ist die: Kann diese oder jene Gewohnheit sich als dem Evangelium gemäß ausweisen? ${ }^{3}$

Es ist klar, dass die oben genannte Frage sich auch auf das Ordensleben, wie wir es führen, ausdehnen muss und sich auch schon längst darauf ausdehnt. Fast alle jungen Menschen, die heute daran denken, sich einer Ordensgemeinschaft anzuschließen, fragen so, und viele schließen sich ihr deshalb nicht an, weil sie glauben, die Frage mit Nein beantworten zu müssen. ${ }^{4}$ In diese Situation hinein spricht das Konzil, und darum sagt es im ersten Satz, dass die genannte Frage trotz aller Einwände grundsätzlich mit Ja zu beantworten ist, d. h., dass das Ordensleben dem Evangelium entspricht, weil es im Wort und im Leben Christi seinen Grund hat. ${ }^{5}$

Wenn wir also sagen: Grundsätzlich gründet das Ordensleben im Wort und im Leben des Herrn, so ist mit dieser Aussage nicht schlechthin unser Ordensleben, wie wir es konkret führen, gebilligt, sondern es ist ein Maßstab an es angelegt, nach dem es sich zu richten hat. Nicht einfachhin alles, was wir bisher

${ }^{1}$ II. Vat. Konzil, Die dogmatische Konstitution über die Kirche Lumen gentium, Nr. 43.

${ }^{2}$ Vgl. Johannes Paul II., Apost. Schreiben Vita consecrata, Bonn 1996, Nr. 14.

3 A. Herzig, Ordens-Christen. Theologie des Ordenslebens in der Zeit nach dem Zweiten Vatikanischen Konzil, Würzburg Echter 1991, S. 399-401.

${ }^{4}$ Vgl. Johannes Paul II., Enzyklika Veritatis splendor, Bonn 1995, Nr. 6.

${ }^{5}$ Vgl. L. Boff, Zeugen Gottes in der Welt. Ordensleben heute, Zürich-Einsiedeln-Köln 1985, S. 37-38. 
gelebt haben, ist deshalb schon als Ordensleben ausgewiesen, sondern es muss daraufhin geprüft werden, ob es Nachfolge Christi ist oder nicht. Denn vielleicht ist manche stoische, mehr vom Ideal der aszetischen Selbstbeherrschung als von der Liebe Christi diktierte Vorstellung in uns, die sich vor dem Richtmaß des Evangeliums nicht halten lässt. ${ }^{6}$

In Wort und Leben des Herrn sind die evangelischen Räte begründet. Warum? Zunächst ist Jesus, so wie ihn die Evangelien uns schildern, arm und ehelos in Palästina umhergewandert, und er hat die Armen seliggepriesen und denen, die es fassen können, etwas darüber gesagt, dass man sich selbst um des Himmelreiches willen irren darf. Er hat weiter unter der Schar seiner Anhänger einige herausgerufen, die er mit sich auf den Weg nahm, sodass sie alles verlassen sollten, nur aufgrund seines Rufes. Sie lebten mit ihm sein Leben. ${ }^{7}$

Diese Gruppe der Jünger Jesu, die mit ihrem Meister lebte, herausgenommen aus den Banden der Familie, die mit ihm für die Verkündigung des Reiches Gottes da war, ist unser Vorbild. In dieser Gruppe finden wir die Wurzeln dessen, was sich später als der viel verzweigte Baum des Ordenslebens in der Kirche entwickelt hat. Der Ruf der Zeit, und das heißt der Ruf des Heiligen Geistes befiehlt uns, heute nach diesen Wurzeln zu schauen und den Zugang zu ihnen freizulegen. ${ }^{8}$

Der Sinn unseres Lebens und unseres Dienstes an der Kirche, also unsere Daseinsberechtigung als Ordenschristen, hängt davon ab, ob uns die Freilegung unserer Wurzeln gelingt. Die Kirchenkonstitution schildert nämlich das Ordensleben folgendermaßen: „Auch die Lebensform, die der Sohn Gottes annahm, als er in die Welt eintrat, um den Willen des Vaters zu tun, und die er den Jüngern, die ihm nachfolgen, vorgelegt hat, ahmt dieser Stand deutlicher nach und bringt sie in der Kirche ständig zur Darstellung. “9 In dieser Aussage dürfen wir keine beruhigende Feststellung, sondern einen aufrüttelnden Ruf, eine positive Kritik sehen. ${ }^{10}$ Ahmt unser Ordensleben, wie wir es konkret führen, die Form des Lebens, das die Jünger in Armut und Ehelosigkeit mit ihrem Meister lebten,

${ }^{6}$ Ch. Schütz OSB, Sendung und Erwartung der Ordensgemeinschaften heute, in: Ordenskorrespondenz, 46 Jhg. 2005, S. 168-169.

${ }^{7}$ Vgl. Vita consecrata, Nr. 18.

${ }^{8}$ Vgl. A. Kraus, Franziskanisches Apostolat im Lichte des II. Vatikanischen Konzils, Rom 1970, S. 34.

9 Lumen gentium, Nr. 44.

${ }^{10}$ Ordens-Christen, S. 21. 
deutlicher nach als ein anderer christlicher Stand $?^{11}$ Gibt unser Leben, geben unsere Gemeinschaften dieser Lebensform in der Kirche ständige Gegenwart? Oder leben wir vielleicht tatsächlich die Armut und Ehelosigkeit nicht so, dass sie den Menschen von heute näher zu Christus führen, müssen wir etwas an dem Gefüge unseres Lebens ändern, damit wir die Aufgabe, die uns von unserer Berufung gestellt ist, richtig erfüllen können ${ }^{12}$

Diese Frage ist nicht nur von Bedeutung für uns selbst und unsere Ausrichtung auf Christus. ${ }^{13}$ Sie ist auch von Bedeutung für die Kirche. Denn unsere Christusnachfolge hat nicht nur den Sinn, für uns selbst Christus in uns auszuprägen, sondern damit zugleich auch den anderen, den Christen und den Nichtchristen, Christi Lebensform vor Augen zu führen. ${ }^{14}$ Dies ist für uns kein Grund zum Sichrühmen, sondern eine uns große Last und Verantwortung. Ebenso wie der hl. Paulus sich die Verkündigung des Evangeliums nicht zum Ruhm anrechnen kann, sondern sagen muss: „denn ein Zwang liegt auf mir. Wehe mir, wenn ich das Evangelium nicht verkünde!"15, so liegt auf uns die Last, Christi Lebensform den Menschen vorzuleben. ${ }^{16}$ Wehe uns, wenn wir uns dieser Verpflichtung entziehen, und sei es auch unter Berufung auf lieb gewordene Gesetze und Gebräuche! Denn die Christen und Nichtchristen, die von uns die Sichtbarmachung des Lebens Christi verlangen können, werden vor Gottes Angesicht unsere Richter sein, wenn wir ihnen verweigert haben, was sie nach dem uns gewordenen Ruf von uns verlangen konnten. ${ }^{17}$ Das Konzil drückt diese Tatsache so aus: „Die Ordensleute sollen sorgfältig darauf achten, dass durch sie die Kirche wirklich von Tag zu Tag mehr den Gläubigen wie den Ungläubigen Christus sichtbar mache, wie er auf dem Berg in der Beschauung weilt, oder wie er den Scharen das Reich Gottes verkündigt, oder wie er die Kranken und Schwachen heilt und die Sünder zum Guten bekehrt, oder wie er die Kinder segnet und allen Wohltaten erweist, immer aber dem Willen des Vaters gehorsam

11 Vgl. Zeugen Gottes in der Welt, S. 37.

12 Vgl. H. Buob, Berufen zur Hingabe. Die evangelischen Räte, Linz Veritatis 1990, S. 11.

13 Ordens-Christen, S. 23.

${ }^{14}$ Vgl. K. Recker, Heute nach dem Evangelium leben, Mainz Matthias-Grünewald 1979, S. 43.

151 Kor 9,16.

16 Veritatis splendor, Nr. 19.

17 Vgl. Berufen zur Hingabe, S. 12. 
ist, der ihn gesandt hat. ${ }^{\text {18 }}$ Wir erkennen an diesem Text, dass kein Ordenschrist, auch der rein kontemplative nicht, sich der Verpflichtung entziehen kann, seinen Mitmenschen das Leben Christi zu vermitteln. Jeder soll es natürlich nach der ihm gewordenen Gestalt der Berufung tun, aber alle Ordenschristen schulden allen Menschen, die sie von außen oder innen erreichen können, die Vermittlung der Lebensform Christi, die Nachfolge Christi. ${ }^{19}$

\section{Wirkkraft des Heiligen Geistes}

Es wäre nun sehr einfach, wenn wir in der „Nachfolge Christi“ ein handhabbares Prinzip, ein Rezept besäßen, das wir nur auf unser Leben anzuwenden hätten, um zum richtigen Ordensleben zu gelangen. So ist es aber gerade nicht. Gewiss sind bestimmte Haltungen im Leben Christi ganz charakteristisch, z. B. die Hingabe an den Willen des Vaters, die Demut, die Bereitschaft zum Dienst, die Liebe zur Armut, die Liebe zu den Sündern, zu den Verzweifelten und Verlassenen. Aber alle diese Haltungen stehen im Leben Christi doch nicht schematisch, sodass wir im Voraus wissen könnten, wie er in einer bestimmten Situation handelt. ${ }^{20}$ Denken wir z. B. an die Perikope, wo Matthäus von der Begegnung Christi mit der kananäischen Frau berichtet. ${ }^{21}$ Wenn wir Christi Liebe zu den Kranken kennen, werden wir annehmen, dass er die Tochter dieser Frau sofort heilt, wie etwa auch den Knecht des Hauptmanns von Kapharnaum, der wie die Frau kein Jude war. Aber Jesus weigert sich zunächst unter Berufung auf den Willen seines Vaters, die Tochter der Frau zu heilen. „Ich bin nur zu den verlorenen Schafen des Hauses Israel gesandt." Wir sehen, dass es in Jesu Leben kein Rezept gibt. Sein Handeln ist überraschend.

Hier liegt für uns ein echtes Problem. Christi Persönlichkeit ist unendlich reich, eben göttlich reich, und dies gerade in seiner menschlichen Gestalt. ${ }^{22}$ Wie können wir ihm da nachfolgen? So oft hören wir den Rat, wir sollten uns in jeder Situation fragen, was Jesus hier wohlgetan hätte. Aber wer von uns kann

${ }^{18}$ Lumen gentium, Nr. 46.

19 Vgl. Hl. Kongregation für Orden und Säkularinstitute, Informatives Dokument, in: AAS (1984), 1734; Vgl. auch Ordens-Christen, S. 30.

${ }^{20}$ Zeugen Gottes in der Welt, S. 40.

${ }^{21}$ Mt 15,21-28.

${ }^{22}$ Vgl. Zeugen Gottes in der Welt, S. 95. 
die Antwort darauf geben? Haben wir das so oft überraschende, nicht in ein Rezept zu fassende Handeln Jesu durchschaut? Keineswegs!

Es ist hier die Stelle, sich daran zu erinnern, dass das Ordensleben nur in der Kraft des Heiligen Geistes in der Nachfolge Christi bleiben kann. ${ }^{23}$ Die natürlich notwendige äußere Regelung des Ordenslebens, die Konstitutionen einer Gemeinschaft helfen dann nicht in die Nachfolge Christi hinein, wenn sich die Herzen nicht der Kraft des Heiligen Geistes öffnen. Dies ist eine Tatsache, die wir vielleicht zu sehr an den Rand unseres Bewusstseins gedrängt haben, doch sie muss wieder in der Mitte stehen. ${ }^{24}$

Gewiss muss eine Gemeinschaft von Ordenschristen eine Lebensregel haben. Das Leben muss sich in einer Ordnung kristallisieren. Aber es ist ein verhängnisvoller Irrtum zu meinen, die Nachfolge des lebendigen Christus' sei schon dann in einer Gemeinschaft gesichert, wenn diese Regel aufs Genaueste erfüllt wird. Christus ist eine lebendige Person, und ihm nachfolgen heißt immer mehr als ein Gesetz erfüllen. Denken wir nur an seine Auseinandersetzungen mit den Pharisäern, etwa über die Sabbatfrage. Betrachten und lieben wir uns in den Geist Christi hinein, und wir werden sehen, dass wir eine viel weitere und mehr elastische Auffassung unserer Konstitutionen haben müssen, wenn wir wirklich Christus nachfolgen wollen. Man kann nämlich in einer bestimmten Situation ein Gesetz erfüllen und trotzdem die Liebe verfehlen. ${ }^{25}$

Nun können wir aber dagegen fragen: Landen wir nicht bei einer zu starken Betonung der Verantwortung des Einzelnen in den verschiedenen Situationen und darauf läuft das Gesagte ja hinaus - in einem Subjektivismus? Besteht nicht die Gefahr, dass dann die Ordensgemeinschaft auseinanderfällt? ${ }^{26}$

Wenn diese Gefahr besteht, dann wegen unseres Kleinglaubens, in dem wir die inneren, geistlichen Kräfte, die eine Gemeinschaft zusammenhalten, zu gering einschätzen. Hier ist nämlich eine Stelle, an der unser Glaube an den Heiligen Geist Wirklichkeit und Macht gewinnen muss. Jedes Gesetz ist nur ein Rahmen, und es muss Raum geben für das Wirken des Geistes in den Herzen. Nur der Heilige Geist kann uns in das lebendige Herz des Herrn einführen, nur er kann die Nachfolge Christi für Menschen möglich machen. Nicht ein Rezept oder ein Gesetz, das wir in der Hand haben und das menschlich handhabbar

\footnotetext{
23 J. M. R. Tillard, Vertrauen zur Gemeinschaft, Freiburg-Basel-Wien Herder 1973, S. 53.

${ }^{24}$ Vgl. Vita consecrata, Nr. 5.

${ }^{25}$ Franziskanisches Apostolat im Lichte des II. Vatikanischen Konzils, S. 37.

${ }^{26}$ Franziskanisches Apostolat im Lichte des II. Vatikanischen Konzils, S. 38.
} 
ist, öffnet uns die Tür zur Nachfolge Christi. Das Gesetz ist nur ein Rahmen, und es soll ein möglichst weiter Rahmen sein, damit der Geist darin seine Wirkkraft entfalten kann. Denn er allein, der Heilige Geist, führt uns in die Nachfolge Christi ein. ${ }^{27}$

Darum sollen die Ordenschristen so erzogen werden, dass sie in Demut und Verantwortlichkeit Entscheidungen fällen können, die ihnen in der Nachfolge Christi zukommen. Nicht Subjektivismus und das Auseinanderfallen der Gemeinschaft, sondern die größere Möglichkeit der Liebe und darum die tiefe, aus den Wurzeln des Glaubens aufsteigende Einheit ist die Frucht des Geistes. Aber diese Frucht kann nur wachsen, wenn sie Raum hat, d. h., wenn wir im Glauben das Wagnis der Offenheit auf uns nehmen. Wenn wir nämlich jede menschlich mögliche Gefahr von vornherein unterdrücken wollen, unterdrücken wir auch die Chancen, die Möglichkeiten des Geistes und machen gerade das unmöglich, was wir wollen, nämlich die Nachfolge Christi, wohlgemerkt: die Nachfolge Christi in der jedes Gesetz übersteigenden Form des Glaubens, der Hoffnung und der Liebe.

Nur wenn wir in diesem Wagnis des Glaubens das Ordensleben auffassen, hat das Konzil die Menschen nicht getäuscht, sondern die Wahrheit bekannt, wenn es sagt: „Der Ordensstand beweist allen Menschen (...) die wunderbare, unbegrenzte Wirkkraft des Heiligen Geistes in der Kirche. "28 Wenn man meint, in der bloßen Befolgung von Konstitutionen bestehe das Ordensleben, so beweist man damit gerade, dass man nicht an die wunderbare, unbegrenzte Wirkkraft des Heiligen Geistes glaubt. Wenn man nicht zugibt, dass der Heilige Geist, wie Christus selbst, überraschend sein kann, dass man sich immer neu auf ihn einstellen muss, dass man manches nicht sofort versteht, was dennoch eine Frucht seines Wirkens ist, dann hat man die Anregungen des Konzils nicht aufgenommen. ${ }^{29}$

Wir dürfen uns also die Erneuerung des Ordenslebens, die dem Konzil vorschwebt, nicht so vorstellen, als ob wir an die Stelle alter Konstitutionen nur Neue zu setzen hätten, im Übrigen aber in der Erfüllung von Gesetzen das Ordensleben bestünde. Es liegt vielmehr im Sinne des Konzils, dass überhaupt die Einstellung zum Gesetz an sich freier und offener wird, nicht in dem Sinne, dass das Gesetz überflüssig würde, wohl aber in dem Sinne, dass es ein weiter

27 Vgl. Vita consecrata, Nr. 19.

28 Lumen gentium, Nr. 44.

29 Vgl. Franziskanisches Apostolat im Lichte des II. Vatikanischen Konzils, S. 39. 
Rahmen ist, innerhalb dessen das Eigentliche, die Nachfolge Christi, in der Wirkkraft des Heiligen Geistes und im Glauben an ihn erst geschieht. ${ }^{30}$

Selbst die Hierarchie der Kirche legt sich in Bezug auf das Ordensleben in der Kirchenkonstitution die Regel auf, dass sie „, in gelehriger Gefolgschaft gegen den Antrieben des Heiligen Geistes" die Ordensgemeinschaften in der Kirche lenken soll und nur so lenken kann. ${ }^{31}$

Warum ist diese Wirkkraft des Heiligen Geistes bei der Betrachtung des Ordenslebens in der Kirche mehr zu beachten als bei anderen Wirklichkeiten? Lassen wir uns hier einen Hinweis geben durch die Kirchenkonstitution: „Ein derartiger Stand ist, in bezug auf die (...) hierarchische Verfassung der Kirche, kein Zwischenstand zwischen dem der Kleriker und dem der Laien. Vielmehr werden aus beiden Gruppen Christgläubige von Gott gerufen, im Leben der Kirche sich einer besonderen Gabe zu erfreuen und, jeder in seiner Weise, für ihre Heilssendung zu nützen. “32

Diese Aussage bedeutet: Wir können den Ordensstand nicht zur hierarchischen Verfassung der Kirche rechnen, er gehört vielmehr zu ihren durch den Heiligen Geist bewirkten charismatischen Lebensäußerungen. ${ }^{33}$ Das Konzil hat daher auch mit Absicht den Ordensstand nicht nach dem dritten und vierten Kapitel der Kirchenkonstitution behandelt, wo von den Amtsträgern und von den Laien die Rede ist, sondern zunächst noch das Kapitel über die Berufung aller Christen zur Heiligkeit folgen lassen. Es wollte schon durch diese Anordnung sagen, dass man unter der Rücksicht der hierarchischen Verfassung nur Amtsträger und Laien unterscheiden kann, dass aber der Ordensstand nur durch die charismatischen Gaben des Heiligen Geistes begründet wird. ${ }^{34}$ Das schließt natürlich nicht aus, dass es sich um einen echten kirchlichen Stand handelt und dass kirchliche Amtsträger in diesen Stand aufnehmen und sie für seine gesunde Entwicklung verantwortlich sind. Man muss sich nur bewusstmachen, dass ein kirchlicher Stand nicht allein durch die hierarchische

${ }^{30}$ J.M.R Tillard, Frei sein in Gott. Zur Praxis des Ordenslebens heute, Freiburg-Basel-Wien Herder 1979, S. 75 u. 78.

31 Lumen gentium, Nr. 45.

32 Lumen gentium, Nr. 43.

${ }^{33}$ Union der Generaloberen, Gottgeweihtes Leben heute: Charismen in der Kirche für die Welt, in: Sekretariat der Deutschen Bischofskonferenz (Hrsg.), Arbeitshilfen 120, Bonn 1993, Nr. 8.

34 Ordens-Christen, S. 136. 
Struktur, sondern auch durch die charismatischen Gaben in der Kirche entstehen kann, und ein solcher Lebensstand wird durch das Ordensleben begründet. ${ }^{35}$

Dies ist etwas sehr Wichtiges. Es kann nicht genug betont werden. Mit dieser Tatsache hängt die andere zusammen, dass das Ordensleben nicht durch ein eigenes Sakrament konstituiert wird, sondern durch eine besondere Berufung, die sich auf der Taufgnade erhebt und durch die Kraft des Heiligen Geistes aus ihr erwächst. ${ }^{36}$ Unter der Rücksicht der hierarchischen Verfassung unterscheiden sich also, innerhalb wie außerhalb der Orden, nur Amtsträger und Laien. Wenn man die Ordenschristen als solche charakterisieren will, muss man eine andere Betrachtungsweise heranziehen, nämlich die der charismatischen Berufung, des Wirkens des Heiligen Geistes, der die Taufgnade in den einzelnen Christen sich in verschiedener Gestalt auswirken lässt. ${ }^{37}$

So sehr also etwa das Priestertum in der Kirche einen geistlichen Stand begründet und auch der Ordensstand ein geistlicher Stand ist, so ist die Wirklichkeit, durch die hier ein Stand begründet wird, doch je verschieden. Beim Priester begründet den Stand das Sakrament der Handauflegung, das zur hierarchischen Verfassung der Kirche gehört. Beim Ordenschristen begründet den Stand die von der Kirche anerkannte charismatische Berufung zu einem Leben nach den evangelischen Räten. Das bedeutet, dass für die Ordenschristen die Wirklichkeit der Berufung eine ausschlaggebende Bedeutung hat. Der engere Jüngerkreis Christi wurde aus der Zahl seiner Anhänger allein durch die Wahl Jesu und durch seinen Ruf ausgesondert. Manche, die ihm nachfolgen wollen, schickt er wieder nach Hause ${ }^{38}$, andere aber, die gar nicht an eine Berufung denken, holt er aus ihrem Lebenskreis heraus, wie etwa Levi, den Zöllner. ${ }^{39}$ Die Frage, die sich die Ordenschristen beim Beginn ihres Ordenslebens stellen müssen, ist also vor allem die nach der Berufung. Diese Frage ist nicht einfachhin durch die Aufnahme durch Ordensobere schon entschieden, da hier eben das Wirken des Heiligen Geistes die entscheidende Rolle spielt, der nicht nur durch das Oberenamt wirkt, sondern auch durch die tausend Umstände des Lebens und durch eine Führung, die sich in der Stimme des Gewissens bemerkbar macht.

${ }^{35}$ Vgl. Gottgeweihtes Leben heute: Charismen in der Kirche für die Welt, Nr. 10.

${ }^{36}$ Vgl. Zeugen Gottes in der Welt, S. 23.

37 Vgl. Vita consecrata, Nr. 14 u. 30.

${ }^{38} \mathrm{Vgl}$. Mk 5,19; siehe auch Mt 8, 19 ff.

$39 \mathrm{Mk} 2,14$. 
Es ist auffallend, wie gerade heute die Gedanken junger Ordenschristen um diese Frage der Berufung kreisen. Dies ist an sich ein gutes Zeichen, und man muss ihnen helfen, indem man mit ihnen ihren Lebensweg durchspricht und mit ihnen zusammen nach Anzeichen für die echte Berufung sucht. ${ }^{40}$ Hierbei ist es notwendig, dass man in der Unterscheidung der Geister zu Hause ist, etwa die Regeln kennt, die der hl. Ignatius als Hilfe für die Lebenswahl vorschlägt. Auch von Psychologie sollte man eine gewisse Kenntnis haben, damit man die allgemein menschlichen Komponenten nicht vorschnell für Antriebe der Gnade ansieht. Auf jeden Fall kommen wir nicht um diese persönliche Beratung der jungen Ordensleute herum, und es ist nur ein Zeichen von Ratlosigkeit, wenn man ihnen sagt: „Ihr wollt ja in allem selbstständig sein; nun fällt also selbst die Entscheidung!“ Ein Rat soll die Entscheidung nicht abnehmen, sondern er soll die richtige Entscheidung erst ermöglichen. Vielleicht versagen hier gerade in der heutigen Umbruchsituation viele Oberen, weil sie nicht genügend darin geschult sind, die Frage der Berufung als die wichtigste Frage des Ordenslebens anzusehen. Das Denken der älteren Generation ist oft mehr an allgemeine Normen als an das Horchen auf die charismatische Berufung gewöhnt. ${ }^{41}$

Hören wir nach dem Gesagten noch einmal die oben zitierte Aussage des Konzils: „Vielmehr werden aus beiden Gruppen [d.h. Kleriker und Laien] Christgläubige von Gott gerufen, im Leben der Kirche sich einer besonderen Gabe zu erfreuen und, jeder in seiner Weise, für ihre Heilssendung zu nützen. “42 Die Ordenschristen werden gerufen, sie erhalten eine besondere Gabe des Heiligen Geistes, damit jeder in seiner Weise für die Heilsmission der Kirche da ist. Wenn das so ist, dann kommt alles darauf an, diesen Ruf festzustellen und zu erkennen, in welche Richtung er weist, damit der Heilige Geist auch wirklich erreichen kann, was er anzielt.

Noch an zwei anderen Stellen der Konstitution, in Nr. 44 und Nr. 47, wird von der Berufung im dargelegten Sinn gesprochen. Jeder soll leben „entsprechend der Gestalt der eigenen Berufung “43, und „jeder, der zum Lebensstand der Räte berufen ist, soll eifrig bemüht sein, in der Berufung, zu der er von Gott

\footnotetext{
${ }^{40}$ Zeugen Gottes in der Welt, S. 30-32.

41 Zeugen Gottes in der Welt, S. 187-188.

${ }^{42}$ Lumen gentium, Nr.43.

${ }^{43}$ Lumen gentium, Nr. 44.
} 
gerufen wurde, zu bleiben. ${ }^{\text {" }}{ }^{4}$ Wir sehen also, dass die Konstitution die Frage der Berufung und das heißt das Wirken des Heiligen Geistes sehr ernst nimmt.

\section{Weg der Liebe}

Die Übernahme der evangelischen Räte in einer Gemeinschaft innerhalb der Kirche hat den Sinn, das Leben der Ordenschristen tiefer in die Nachfolge Christi zu führen, und zwar geschieht das letztlich in der Kraft des Heiligen Geistes. Doch müssen wir noch fragen, in welcher Haltung sich die Christusnachfolge konkret auswirkt und woran man nun das Wirken des Heiligen Geistes erkennen kann, d. h. welches die Frucht des Geistes ist. Mit dem Konzil können wir diese Frage mit einem Wort beantworten: Die Frucht des Geistes, die allein ein Leben nach den evangelischen Räten rechtfertigt und sinnvoll macht, ist die Liebe. Wo die Liebe Christi wächst, dort erfüllt das Leben nach den evangelischen Räten seinen Sinn, und nur dort. ${ }^{45}$

Diese Aussage findet sich in Nr. 44 der Konstitution, wenn es dort heißt, dass die Räte „zur Liebe führen“, oder Nr. 45, wo gesagt ist, dass durch die Räte „die (...) Liebe zu Gott und den Nächsten einzigartig gefördert wird“. Hier dürfen wir wie bisher diese Aussagen nicht als beruhigende Feststellungen, sondern als Forderungen verstehen, nach denen wir unser Leben überprüfen müssen. Nur dort, wo tatsächlich durch die Betrachtung der evangelischen Räte die Liebe zu Gott und den Menschen wächst, werden die Räte christlich gelebt. ${ }^{46}$ Dort, wo ein Eheloser statt größerer Liebe zu den Menschen von Jahr zu Jahr ein immer verbitterteres und verdrehteres Wesen zeigt, wird der Rat der Ehelosigkeit eben nicht gelebt, so sehr vielleicht auch in sexueller Hinsicht diesem Menschen nichts vorzuwerfen ist. Vielleicht liegt in einem solchen Fall gar keine echte charismatische Berufung vor, oder die Gemeinschaft und die Arbeit überfordern den Menschen, geben ihm zu wenig Rückhalt, sodass seine Berufung sich nicht entfalten kann. ${ }^{47}$

Prüfen und fragen wir uns also, ob die Praxis der Räte Christi, wie wir sie leben, wirklich so ist, dass, mit den Worten der Konstitution zu sprechen, „die

\footnotetext{
${ }^{44}$ Lumen gentium, Nr. 47.

${ }^{45}$ Vgl. Benedikt XVI., Enzyklika Deus caritas est., Bonn 2005, Nr. 20.

${ }^{46}$ Vgl. Veritatis splendor, Nr. 20.

47 Vgl. Johannes Paul II, Apost. Schreiben Novo millennio ineunte, Bonn 2001, Nr. 49.
} 
Räte (...) zur Reinigung des Herzens beitragen und ständig die Glut der Liebe anfachen. ${ }^{\text {"48 }}$

Aus den Worten Christi ist uns allen klar, dass die hier gemeinte Liebe zugleich Gottes- und Nächstenliebe ist, denn das zweite Gebot ist dem ersten gleich, und Christus identifiziert sich bei Mt 25 ausdrücklich mit allen Armen und Bedrückten. Er identifiziert sich mit allen Armen und Bedrückten, also nicht nur mit den uns Nahestehenden, nicht nur mit denen, die wir verstehen, nicht nur mit denen, die Gott lieben, sondern gerade auch mit den Sündern..$^{49}$ Die Liebe zum Sünder und die Liebe zu unserem Feind wird immer wieder der Prüfstein sein, an dem wir erkennen können, ob die Übernahme der evangelischen Räte bei uns wirklich die Frucht des Heiligen Geistes zum Wachsen gebracht hat. „Wenn ihr nämlich nur die liebt, die euch lieben, welchen Lohn könnt ihr dafür erwarten? (...) Und wenn ihr nur eure Brüder grüßt, was tut ihr damit Besonderes?"50

Daraus ergibt sich: Auf keinen Fall dürfen die Ordensgemeinschaften abgeschlossene geistige Räume bleiben, sozusagen Zirkel, von denen man annimmt, sie kümmerten sich nur um ihre Sache und um diejenigen, die mit ihnen einer Meinung sind. Und zwar fordert nicht einmal in erster Linie der Dialog der Kirche mit der Welt, von dem heute so viel gesprochen wird, sondern die Liebe Christi und ihre drängende Kraft selbst die Öffnung zu allen. Nicht weil die Welt auf uns wartet - denn es ist eine Frage, ob sie das tut -, sondern weil Christus durch uns zu den Menschen kommen will, muss sich in der Liebe zu allen das Leben nach den evangelischen Räten bewähren.

Die Liebe Christi hat eben eine universale Sprengkraft, sie zerreißt alle Enge, allen Parteigeist, alle Selbstsucht der einzelnen Ordensgemeinschaften, allen Gruppenegoismus, und nur dann ist sie wirklich anwesend, wenn sie diese Wirkung tatsächlich zeigt.

\section{Geheimnis der Kirche}

Damit hängt nun noch eine letzte Aussage der Kirchenkonstitution zusammen. Hören wir sie im Wortlaut: „Weil aber die evangelischen Räte ihre Befolger durch

\footnotetext{
${ }^{48}$ Lumen gentium, Nr. 46.

49 Berufen zur Hingabe, S. 143.

50 Mt 5, 46 ff.; Vgl. Franziskanisches Apostolat im Lichte des II. Vatikanischen Konzils, S. 41.
} 
die Liebe, zu der sie hinführen, auch in besonderer Weise mit der Kirche und ihrem Geheimnis verbinden, muß ihr geistliches Leben auch dem Wohl der ganzen Kirche gewidmet sein. Daraus ergibt sich die Pflicht, nach Kräften und entsprechend der Gestalt der eigenen Berufung, durch Gebet oder auch tätiges Wirken sich um die Einwurzelung und Festigung des Reiches Christi in den Seelen und seine weltweite Ausbreitung zu bemühen. Deshalb auch schützt und fördert die Kirche den eigenen Charakter der verschiedenen Ordensinstitute. So erscheint das Bekenntnis zu den evangelischen Räten als ein Zeichen, das alle Glieder der Kirche wirksam zur eifrigen Erfüllung der Pflichten ihrer christlichen Berufung hinziehen kann und soll. ${ }^{\text {" } 11}$

Das Ordensleben drückt also das Geheimnis der Kirche in besonderer Weise aus, und zwar - das ist zu betonen - durch die Liebe, zu der es hinführt. Das heißt in dem Maße die Glieder einer Ordensgemeinschaft einander in Liebe tragen und stützen, stellt diese Gemeinschaft die Kirche dar, kommt Christi Lebenshingabe für uns, aus welcher die Kirche sich entfaltet, in dieser kleinen Gemeinschaft sichtbar zum Zuge. ${ }^{52}$ Das bedeutet aber zugleich, dass sich dieselbe Liebe Christi, in der er sich für uns geopfert hat, in der Öffnung dieser Ordensgemeinschaft für die anderen Menschen, für die Sünder, die Armen und Schwachen, zeigen muss. Auch das ist Geheimnis der Kirche. Denn letztlich ist das Geheimnis der Kirche nichts anderes als das „für uns“ des sich opfernden Christus. Darum entspringt aus seiner geöffneten Seite am Kreuz die Kirche. Überall dort, wo diese Liebe in einer Ordensgemeinschaft sichtbar wird, nach innen und nach außen, ist das Geheimnis der Kirche anwesend. ${ }^{53}$

Es ist hier noch einmal darauf hinzuweisen, dass das Geheimnis der Kirche und d. h. die Liebe Christi in den verschiedenen Berufungen der Ordenschristen immer ganz anwesend ist, also als Gottes- und Nächstenliebe. ${ }^{54}$ Man kann daher die kontemplative Berufung und die apostolische oder karitative Berufung innerhalb der Berufung zum Ordensleben nicht so aufspalten, als wäre die eine ausschließlich oder mehr Liebe zu Gott und die andere mehr Liebe zu den Menschen. ${ }^{55}$ Diese falsche Vorstellung stammt aus der im Mittelalter üblichen, exegetisch unhaltbaren Interpretation von Lk 10, 38-42. Da diese Perikope im

${ }^{51}$ Franziskanisches Apostolat im Lichte des II. Vatikanischen Konzils, Nr. 44.

52 Vgl. Berufen zur Hingabe, S. 46.

53 Vgl. Vita consecrata, Nr. 29.

54 Veritatis splendor, Nr. 14.

${ }^{55}$ Vgl. Zeugen Gottes in der Welt, S. 35-36. 
Dekret über die zeitgemäße Erneuerung des Ordenslebens in Nr.5 herangezogen wird, so ist es sicherlich nützlich, dass wir uns etwas näher mit ihr befassen. Hören wir erst die ganze Perikope: „Sie zogen zusammen weiter und er kam in ein Dorf. Eine Frau namens Marta nahm ihn auf. Sie hatte eine Schwester, die Maria hieß. Maria setzte sich dem Herrn zu Füßen und hörte seinen Worten zu. Martha aber war ganz davon in Anspruch genommen für ihn zu sorgen. Sie kam zu ihm und sagte: Herr, kümmert es dich nicht, dass meine Schwester die ganze Arbeit mir allein überlässt? Sag ihr doch, sie soll mir helfen! Der Herr antwortete: Marta, Marta, du machst dir Sorge und Mühen. Aber nur eines ist notwendig. Maria hat das Bessere gewählt, das soll ihr nicht genommen werden. ${ }^{\text {"56 }}$

Fangen wir mit der Erklärung bei der Aussage Jesu an, dass Maria das Bessere erwählt hat. Es heißt nicht „das Bessere“, als ob auch Marta etwas Gutes, wenn auch etwas weniger Gutes erwählt hätte. Nein, es geht nicht um das „mehr oder weniger" Gute, sondern um das, was in der Situation richtig und was falsch ist. Maria tut das Richtige, Marta das Falsche. ${ }^{57}$

Wenn wir die Perikope verstehen wollen, dürfen wir nicht die Tätigkeiten der beiden Schwestern an sich betrachten, sondern in Bezug auf den Herrn. Nachfolge Christi heißt: Auf Christus schauen, tun, was sich aus seinem Tun ergibt. Wenn Christus aber lehrt, dann ergibt sich für den Jünger daraus das Hören. Maria lässt sich vom Herrn bestimmen. Sie hört, wenn er lehrt. Marta aber lässt sich von ihren eigenen Ideen bestimmen. Sie erfasst die Situation nicht, sie tut nicht, was durch das Tun des Herrn in der Situation von ihr gefordert ist.

Maria wird auch, wenn die Zeit dazu da ist, dienen. Jetzt aber, da der Herr lehrt, muss sie hören. Der Unterschied zwischen den beiden Schwestern liegt also nicht in Kontemplation und Aktion, sondern darin, dass die eine in der Nachfolge Christi steht und nach dem Herrn schaut, die andere nach ihrem eigenen Gutdünken handelt. Und Christus sagt nicht: Marta tut etwas Gutes, Maria etwas Besseres, sondern er tadelt Marta und sagt doch damit: Marta handelt in der Situation falsch, Maria aber richtig, denn eines nur ist notwendig, nämlich auf mich zu schauen und mir nachzufolgen. Wenn ich also lehre, muss man hören, wenn ich sage: „Dienet einander!“, muss man dienen. ${ }^{58}$

56 Lk 10, 38-42.

57 Vgl. Vita consecrata, Nr. 28.

58 Vgl. Benedikt XVI., Predigt des Heiligen Vaters am 11. September 2006, in: Sekretariat der Deutschen Bischofskonferenz (Hrsg.), Verlautbarungen des Apostolischen Stuhls 174, Bonn 2006, 52. 
Der ganze Unterschied liegt also in der Christusbeziehung, nicht in den verschiedenen Tätigkeiten der beiden Schwestern. Die Zerstreuung der Marta ist nicht in ihrem Tun, sondern in der inneren Zerstreuung ihres Geistes begründet. Sie ist nicht auf das "eine Notwendige" konzentriert. Man kann aber auch gesammelt sein, wenn man in der entsprechenden Situation durch äußere Tätigkeit dient.

Ebenso verhält es sich mit den verschiedenen Berufungen in der Kirche. Jeder Ordenschrist, jede Ordensgemeinschaft wird von Christus anders gerufen. Vor allem gibt es die Verschiedenheit zwischen kontemplativen, apostolischen, karitativen Berufungen. Aber bei jeder Berufung geht es, unter der je verschiedenen Gestalt, um das eine Notwendige, um die Nachfolge Christi, und jedes Mal wird in der Gestalt der je eigenen Berufung die eine Liebe, die zugleich Gottes- und Nächstenliebe ist, verwirklicht. Der Kontemplative verwirklicht durch sein stellvertretendes Gebet ebenso die Nächstenliebe wie der Aktive durch seinen Dienst an den Menschen in diesen Christus selbst erreicht. Daher sagt das Dekret über die zeitgemäße Erneuerung des Ordenslebens: „Inmitten der Vielfalt von Gnadengaben weihen sich alle, die von Gott zum Leben der evangelischen Räte berufen werden und dieses aufrichtig geloben, in besonderer Weise dem Herrn, indem sie Christus nachfolgen, der selbst jungfräulich und arm gelebt und durch seinen Gehorsam bis zum Tod am Kreuz die Menschen erlöst und geheiligt hat. Von der Liebe gedrängt, die der Heilige Geist in ihre Herzen ausgegossen hat, leben sie mehr und mehr für Christus und seinen Leib, die Kirche. Je inniger sie also durch solche Selbsthingabe, die das ganze Leben umfaßt, mit Christus vereinigt werden, desto reicher wird das Leben der Kirche und desto fruchtbarer deren Apostolat. ${ }^{\text {“59 }}$

Doch damit haben wir schon über die Kirchenkonstitution hinausgegriffen. Diese allerdings sagt im Grunde nichts anderes, wenn sie erklärt: „Denn, wenn sie [die Ordensleute] auch zuweilen mit ihren Zeitgenossen nicht in unmittelbarer Weise hilfreich sind, haben sie diese doch auf tiefere Weise in der Liebe Christi gegenwärtig und wirken geistlich mit ihnen zusammen, dass der Bau der irdischen Gesellschaft immer in Gott gründe und auf ihn ausgerichtet sei und seine Erbauer nicht vergeblich arbeiten. ${ }^{\text {"60 }}$

${ }^{59}$ II. Vat. Konzil, Dekret über die zeitgemäße Erneuerung des Ordenslebens, Perfectae caritatis, Nr. 1.

${ }^{60}$ Lumen gentium, Nr. 46. 
Aus der Verbindung mit dem Geheimnis der Kirche ergibt sich nun noch weiter, dass die Orden der kirchlichen Hierarchie, also entweder den Bischöfen oder direkt dem Papst, unterstehen, allerdings so, dass die kirchliche Hierarchie ihrerseits verpflichtet ist, den charismatischen Charakter und damit den eigentlichen Sinn des Ordenslebens zu respektieren und die Ordensleute nicht als billige Arbeitskräfte in einem sinnentfremdeten Bereich auszunutzen. In den Ausführungsbestimmungen zum Dekret Christus Dominus wird der Bischof darauf aufmerksam gemacht, dass er die spezifische Eigenart der einzelnen Orden beachten muss: „(...), wobei die Eigenart eines jeden Verbandes zu berücksichtigen ist. ${ }^{\text {"61 }}$ Hier liegt auch eine unserer Verpflichtungen, nämlich der christlichen Öffentlichkeit und vor allem den Bischöfen ein richtiges Bild vom Ziel des Ordenslebens zu vermitteln. In der Kirchenkonstitution heißt es: „Da die kirchliche Hierarchie die Aufgabe hat, das Volk Gottes zu leiten und auf reiche Weide zu führen, ist sie dafür zuständig, die Übung der evangelischen Räte (...) zu lenken. “" "Auf reiche Weide führen" heißt doch sicher: Im Sinn der Berufung, unter Berücksichtigung des eigentlichen Wesens der Orden ihnen Möglichkeiten zur Entfaltung und zum Dienst am Reiche Gottes geben. Diese Aufgabe haben also die Bischöfe. Umgekehrt sollen die Ordensleute als zum Dienst an der Kirche bestellte Menschen innerhalb der eigenen Berufung den Bischöfen dienen.

Ein letzter Charakterzug der kirchlichen Aufgabe der Orden soll schließlich noch erwähnt werden. Die Christen in der Kirche stehen dauernd in der Spannung „nicht von der Welt, aber in der Welt“. Sie sind unterwegs auf ein endgültiges, verborgen schon anwesendes Ziel zu, müssen aber auf diesem Wege die Gegebenheiten eben dieses Weges und damit die Welt ganz ernst nehmen. Bei der Gebrechlichkeit des erbsündigen Menschen besteht nun immer die Gefahr, dass sogar der Christ sich in seinen weltlichen Aufgaben so einrichtet, dass er vergisst, zu welchem Ziel er unterwegs ist. Dadurch, dass die Ordensleute aus den Beziehungen der Familie und der familiären Ordnung um des Himmelreiches willen herausgerufen sind, können sie in der Kirche der Stachel sein, der immer wieder auf das endgültige Ziel hinweist, der immer wieder daran erinnert, dass der Mensch ein Pilger ist. Dieser Hinweis auf die künftige, endgültige Gestalt aller Dinge, auf das Reich Gottes, wird hier existenziell, d.h. er prägt das gesamte

${ }^{61}$ II. Vat. Konzil, Das Dekret über die Hirtenaufgabe der Bischöfe in der Kirche Christus dominus, Nr. 33.

${ }^{62}$ Lumen gentium, Nr. 45. 
Leben oder soll es prägen, und so kann er ein umso eindringlicheres Zeugnis sein. Allerdings muss dieses Zeugnis echt, also wirklichkeitsnah gelebt werden, in Formen, die die Menschen der jeweiligen Zeit verstehen. Sonst wäre es kein Zeugnis. ${ }^{63}$

Nach den Worten der Konstitution hat „das Volk Gottes (...) hier keine bleibende Heimstatt, sondern sucht die zukünftige. Deshalb macht der Ordensstand, der seine Glieder von den irdischen Sorgen mehr befreit, mehr die himmlischen Güter, die schon in dieser Zeit gegenwärtig sind, auch allen Gläubigen kund, bezeugt das neue und ewige, in der Erlösung Christi erworbene Leben und kündigt die zukünftige Auferstehung und Herrlichkeit

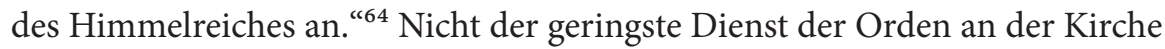
besteht also darin, dass sie immer wieder aus der Geruhsamkeit, in der sich die Christen in der Welt die bleibende Wohnstatt einzurichten versucht sind, herausreißen und Mahner dazu sein wollen, dass jeder Christ sich nur als Pilger und Fremdling richtig versteht.

Mit dem Gesagten hätten wir wohl die Grundzüge der Spiritualität des Ordenslebens herausgestellt, die das sechste Kapitel der Kirchenkonstitution kennzeichnet. Fassen wir die einzelnen Aussagen noch einmal kurz zusammen: 1. Das Ordensleben ist Nachfolge Christi. 2. Diese Nachfolge ist charismatischer Art, also nur in der Kraft und Anregung des Heiligen Geistes, in einer Berufung, vollziehbar. 3. Die Frucht des Wirkens des Heiligen Geistes und damit der Vollzug der Nachfolge Christi ist die Liebe, die, bei aller Verschiedenheit der Gaben und Berufungen, immer Gottes- und Nächstenliebe zugleich ist. 4. Durch diese Liebe, die vom Heiligen Geist gewirkt ist, erweist sich das Ordensleben als ein Geschenk Christi an die Kirche, steht daher mitten im Leben der Kirche und dient ihr, nicht nur durch äußeren Gehorsam und äußere Arbeit, sondern tiefer durch das gelebte Zeugnis, das vor allem die Pilgerschaft der Christen und die Hoffnung auf das künftige Leben bezeugt.

\section{Bibliography}

Dokumente des II. Vatikanischen Konzils:

- Die dogmatische Konstitution über die Kirche, Lumen gentium.

${ }^{63}$ Vgl. Ordens-Christen, S. 34.

${ }^{64}$ Lumen gentium, Nr. 44. 
- Dekret über die zeitgemäße Erneuerung des Ordenslebens, Perfectae caritatis.

- Das Dekret über die Hirtenaufgabe der Bischöfe in der Kirche, Christus dominus.

Benedikt XVI., Enzyklika Deus caritas est., Bonn 2005.

Benedikt XVI., Predigt des Heiligen Vaters am 11. September 2006. In: Sekretariat der Deutschen Bischofskonferenz (Hrsg.), Verlautbarungen des Apostolischen Stuhls 174, Bonn, 2006.

Boff L., Zeugen Gottes in der Welt. Ordensleben heute, Zürich-Einsiedeln-Köln 1985.

Buob H., Berufen zur Hingabe. Die evangelischen Räte, Linz 1990.

Heilige Kongregation für Orden und Säkularinstitute, Informatives Dokument, in: AAS (1984).

Herzig A., Ordens-Christen. Theologie des Ordenslebens in der Zeit nach dem Zweiten Vatikanischen Konzil, Würzburg Echter 1991.

Johannes Paul II, Apostolisches Schreiben Novo millennio ineunte, Bonn 2001.

Johannes Paul II., Apostolisches Schreiben Vita consecrata, Bonn 1996.

Johannes Paul II., Enzyklika Veritatis splendor, Bonn 1995.

Kraus A., Franziskanisches Apostolat im Lichte des II. Vatikanischen Konzils, Rom 1970.

Recker K., Heute nach dem Evangelium leben. Mainz Matthias-Grünewald 1979.

Schütz Ch., Sendung und Erwartung der Ordensgemeinschaften heute, in: Ordenskorrespondenz, 46 Jhg. 2005.

Tillard J.M.R., Vertrauen zur Gemeinschaft, Freiburg-Basel-Wien Herder 1973.

Tillard J.M.R, Frei sein in Gott. Zur Praxis des Ordenslebens heute, Freiburg-Basel-Wien Herder 1979.

Union der Generaloberen, Gottgeweihtes Leben heute: Charismen in der Kirche für die Welt, in: Sekretariat der Deutschen Bischofskonferenz (Hrsg.), Arbeitshilfen 120, Bonn 1993. 